\title{
THE MATTER OF DECISION-MAKING CONTROL OVER OPERATION PROCESSES OF MARINE POWER PLANT SYSTEMS WITH THE USE OF THEIR MODELS IN THE FORM OF SEMI-MARKOV DECISION-MAKING PROCESSES
}

Jerzy Girtler*

Jacek Rudnicki

Gdańsk University of Technology, Poland

*Corresponding author: jgirtl@pg.edu.pl (J. Girtler)

\begin{abstract}
The article presents the possibility to control the real operation process of an arbitrary device installed in the marine power plant based on the four-state semi-Markov process, being the model of the process, which describes the transition process of operational states of the device $\left(e_{k}, k=1,2,3,4\right)$, and the transition process of its technical states $\left(s_{p}, l=1,2,3\right)$. The operational states $e_{k}(k=1,2,3,4)$ have the following interpretation: $e_{1}$-active operation state resulting from the task performed by the device, $e_{2}$-state of ready-to-operate stop of the device, $e_{3}$-state of planned preventive service of the device, $e_{4}$-state of unplanned service of the device, forced by its damage. Whereas the interpretation of the technical states $s_{l}(l=1,2,3)$ is as follows: $s_{1}$ - state of full serviceability of the device, $s_{2}$-state of partial serviceability of the device, and $s_{3}$ - state of unserviceability of the device. All these states are precisely defined for the ship main engine (SG). A hypothesis is proposed which justifies the use of this model to examine real state transitions in marine power plant device operation processes. The article shows the possibility to make operating decisions ensuring a rational course of the device operation process when the proposed model of this process and the dynamic programming method based on the Bellman's principle of optimality are applied. The optimisation criterion adopted when making operating decisions is the expected profit to be gained as a result of functioning of the device in the time interval $\left[\tau_{0}\right.$, $\left.\tau_{m}\right]$, being the sum of the expected profit gained in interval $\left[\tau_{0}, \tau_{1}\right]$ and to be gained in interval $\left[\tau_{1}, \tau_{m}\right]$.
\end{abstract}

Keywords: semi-Markov model, ship main engine, operation process, stochastic process, operational state, technical state, marine power plant equipment

\section{INTRODUCTION}

In Ref. [2], the author presents a possibility to apply the Bayesian decision-making theory for making one of two possible operating decisions when the limiting distribution of the three-state semi-Markov model of technical state transitions of the ship main engine (SG) is known. The article points out that the application of the Bayesian decisionmaking theory and the theory of semi-Markov processes provides an opportunity to make a choice between one of the two following decisions:

- decision $d_{1}$ - first perform the relevant preventive service of the engine to retore its state required for performing the commissioned task, and then start performing the task within the time limit agreed by the customer,

- decision $d_{2}$ - omit the preventive service and start performing the commissioned task.

The engine user must always make one of these two decisions prior to ship's departure. In those situations, the decision-making procedure described in $[2,6,11,12]$ can be very helpful. Making a choice between these two decisions is also of high importance for other marine power plant devices. However, there are more decisions to be made in operating practice. In general, it is important that the decision made ensures that the course of the operation process of not only the 
main engine but also of the remaining devices in the marine power plant is rational (preferably optimal). For an arbitrary marine power plant device, its operation process needs to be controlled. Each of these processes comprises a sequence of casually related states $z_{i} \in Z(i=1,2,3,4)$, bearing the name of operation process states and describing the simultaneous occurrence of operational states $e_{k} \in E$ and technical states $s_{l} \in$ $S$, in which the device can stay $[2,4,5,6,10,12]$. The definitions of states $z_{i} \in Z(i=1,2,3,4), e_{k}(k=1,2,3,4)$, and $s_{l}(l=1,2,3)$ are given in Chapter 2. Their interpretation is similar to that adopted for marine diesel engines $[2,5,6]$.

The need to control the operation process of each device (not only the main engine) installed in the marine power plant by controlling its states $z_{i} \in Z$ results from the fact that during ship sailing, these devices should permanently stay in state $z_{1}$ $=\left(e_{1}, s_{1}\right)$, i.e. simultaneously in state $e_{1}$ of active operation, and state $s_{1}$ of full serviceability. Unfortunately, the wear of a device can lead to its failure, which should be avoided during ship's voyage. When any of marine power plant devices gets damaged during the storm, this leads at least to a complicated and troublesome situation in ship sailing, if not to the emergency situation [11]. In particular, the damage of such an important device as the main engine leads, as a rule, to a catastrophic situation [11]. Those situations, which most often result in ship's sinking, frequently with crew and passengers, can be avoided by applying a proper diagnosing system $(S D G)$ to each device to monitor its technical condition $[2,3,5,7,10,17,18]$. This way, the times of preventive services could be rationally planned and executed to avoid possible failures during ship's voyage. When the preventive service is performed, the device is in state $z_{3}=\left(e_{3}, s_{2}\right)$, being the consequence of simultaneous occurrence of state $e_{3}$ of its preventive service, and state $s_{2}$ of its partial serviceability. However, despite the use of $S D G$, the device can get damaged, and additional unplanned service caused by this damage is required. During this service the device is in state $z_{4}=\left(e_{4}, s_{3}\right)$, which means that it is simultaneously in state $e_{4}$ of unplanned service, and in state $s_{3}$ of unserviceability. Certainly, the device can also be in state $z_{2}=\left(e_{2}, s_{1}\right)$, which occurs when it is simultaneously in the ready-to-operate stop state $e_{2}$, and the full serviceability state $s_{1}$ which allows the device to perform tasks for which it was intended in the design and production phases. Making decision $d_{i}$ that the device should be in state $z$ $(i=1,2,3,4)$ will entail certain financial consequences. These consequences can be expressed as the profit, which takes into consideration both financial gains obtained when the device performs its task and the cost of the device staying in one of those states. The marine power plant user is interested in making a decision which will bring him the profit as large as possible, preferably the maximum. This is possible when using the decision-making (control) theory of semi-Markov processes for making decisions ensuring the maximum profit. These decisions create an optimum strategy, which can be determined using the dynamic programming method based on the Bellman's principle of optimality, or the iterative Howard algorithm $[12,13,15,16,21]$. In the monograph [12], the author describes in detail the issue of decision-making based control of the operation process of internal combustion engines used as ship main engines, which makes use of the process model in the form of six-state semi-Markov process and Howard algorithm. With some adaptations, the proposed method can be used for an arbitrary device in an arbitrary marine power plant. However, a simpler model can also be used for this purpose $[7,10,11,12]$. This article describes the possibility of decision-making based control of the operation process of a marine power plant device which makes use of the process model in the form of four-state semi-Markov process, along with dynamic programming and the Bellman's principle of optimality.

\section{FORMULATING THE OPTIMISATION PROBLEM FOR THE MARINE POWER PLANT DEVICE OPERATION PROCESS BASED ON THE FOUR-STATE SEMI- MARKOV MODEL OF THIS PROCESS AS AN EXAMPLE}

At an arbitrary time $t$ of its operation, the marine power plant device, (the main or auxiliary internal combustion engine, compressor, pump, steam or water boiler, water cooler, or electric motor, etc.), can be in one of four operation process states $z_{i}\left(z_{i} \in Z, i=1,2,3,4\right)$. The operation process states $z_{i} \in Z$ describe the simultaneous occurrence of operational states $e_{k}(k=1,2,3,4)$ and technical states $s_{l}(l=1,2,3)$. Formally, the states $z_{i} \in Z$ can be defined as: $z_{1}=\left(e_{1}, s_{1}\right), z_{2}=\left(e_{2}, s_{1}\right)$, $z_{3}=\left(e_{3}, s_{2}\right), z_{4}=\left(e_{4}, s_{3}\right)$. The operational states $e_{k}$ belong to the set of operational states, $E\left(e_{k} \in E, k=1,2,3,4\right)$, defined by the relation $(1)[10,11,12]$ :

$$
E=\left\{e_{1}, e_{2}, e_{3}, e_{4}\right\}
$$

having the following interpretation:

- $\quad$ state $e_{1}$ resulting from performing the intended task by the marine power plant device,

- $\quad$ state $e_{2}$ resulting from the ready-to-operate stop of the device waiting for start and transition to state $e_{1}$,

- $\quad$ state $e_{3}$ of planned (preventive) service of the device, performed to avoid its possible damage during state $e_{1}$,

- $\quad$ state $e_{4}$ of unplanned service of the device, forced by its damage.

The set $E=\left\{e_{1}, e_{2}, e_{3}, e_{4}\right\}$ of operational states can be considered the set of values of the stochastic process $\{X(t): t \geq 0\}$ with realisations constant in intervals and continuous on the right $[10,12]$.

In the phase of rational operation of all marine power plant devices, it is required that the operational state el takes place only when the device is in the full serviceability state $\left(s_{1}\right)$. Moreover, the existence of state $e_{2}$ is only permissible in this operation when the device is in state s1. When the device is in the partial serviceability state, $\left(s_{2}\right)$, it must get preventive service to avoid its possible damage. Starting this service means the appearance of state $e_{3}$. If the damage cannot be prevented and takes place, the device changes to state $s_{3}$. In 
this case, the service forced by the damage is to be performed, during which the device changes to state $e_{4}$.

The technical states $s_{l}(l=1,2,3)$ being the condition for the existence of states $e_{k}(k=1,2,3,4)$ belong to the set of technical states, $S\left(s_{l} \in S, 1=1,2,3\right)$, defined by the relation (2) $[5,6,10,11,12]$ :

$$
S=\left\{s_{1}, s_{2}, s_{3}\right\}
$$

having the following interpretation:

- state of full serviceability, $s_{1}$, which enables the device to operate in all conditions (in the case of the main engine in the entire load range), for which it was intended in the design and production phases;

- stan of partial serviceability, $s_{2}$, which enables the device to operate in limited conditions, (in the case of the main engine - in the smaller load range), compared to those for which it was intended in the design and production phases,

- $\quad$ state of unserviceability, $s_{3}$, which precludes the device operation (ship moving by the main engine) as intended due to its damage.

The set $S=\left\{s_{1}, s_{2}, s_{3}\right\}$ of technical states can be considered the set of values of the stochastic process $\{W(t): t \geq 0\}$ with realisations constant in intervals and continuous on the right $[2,5,6,12,13,14]$.

Consequently, the states $z_{i}$ belong to the set $Z$ of operation process states defined by the relation (3):

$$
Z=\left\{z_{1}, z_{2}, z_{3}, z_{4}\right\}
$$

having the following interpretation:

- the operation process state $z_{1}=\left(e_{1}, s_{1}\right)$, which exists when the device is in full serviceability state $\left(s_{1}\right)$ and is operated as intended (actively used, or working), which means that it is in operational state $e_{1}$,

- the operation process state $z_{2}=\left(e_{2}, s_{1}\right)$, which exists when the device is in full serviceability state $\left(s_{1}\right)$ and is used passively (not actively: not working) waiting for start, which means that it is in operational state $e_{2}$,

- the operation process state $z_{3}=\left(e_{3}, s_{2}\right)$, which exists when the device is in partial serviceability state $\left(s_{2}\right)$ and for this reason gets preventive service, which means that it is in operational state $e_{3}$,

- the operation process state $z_{4}=\left(e_{4}, s_{3}\right)$, which exists when the device is in unserviceability state $\left(s_{3}\right)$ due to its damage, and for this reason gets unplanned service, which means that it is in operational state $e_{4}$,

A more detailed interpretation of operational states $e_{k}(k=1$, $2,3,4)$ of the marine power plant device was already given when discussing relation (1), while that of technical states $s_{l}$ $(l=1,2,3)$ - for relation (2).

The above interpretation of states $z_{i}(i=1,2,3,4)$ is fairly general, but it can be defined more precisely for an individual device: main or auxiliary internal combustion engine, steam or water boiler, impeller or positive displacement pump, radial or axial compressor, water or oil or air cooler, oil or water or fuel heater, etc.
The set of operation process states of an arbitrary marine power plant device, $Z=\left\{z_{1}, z_{2}, z_{3}, z_{4}\right\}$, can be considered the set of values of the stochastic process $\{Y(t): t \geq 0\}$ with realisations constant in intervals and continuous on the right $[5,7,9,10,11,12]$. This process includes the stochastic process $\{X(t): t \geq 0\}$, with operational states $e_{k}(k=1,2,3,4)$ as its values, and the stochastic process $\{W(t): t \geq 0\}$, with technical states $s_{l}(l=1,2,3)$ of the device as its values.

The graph of state transitions for the process $\{Y(t): t \geq 0\}$ is shown in Fig. 1. This graph has been created based on situations observed in operational practice of each marine power plant device. The states $z_{1}$ and $z_{2}$ are desirable states, while states $z_{3}$ and $z_{4}$ are undesirable, as they make it more difficult to use the power plant device as intended at arbitrary time $t$ of its operation. States $z_{3}$ and $z_{4}$ are unavoidable evil and cannot be escaped in the operation of any device $[9,11$, $12,13,17,18,24]$, but proper control of its operation (such as proper decision-making, for instance) can reduce negative effect of those states on operating parameters, including ship profitability.

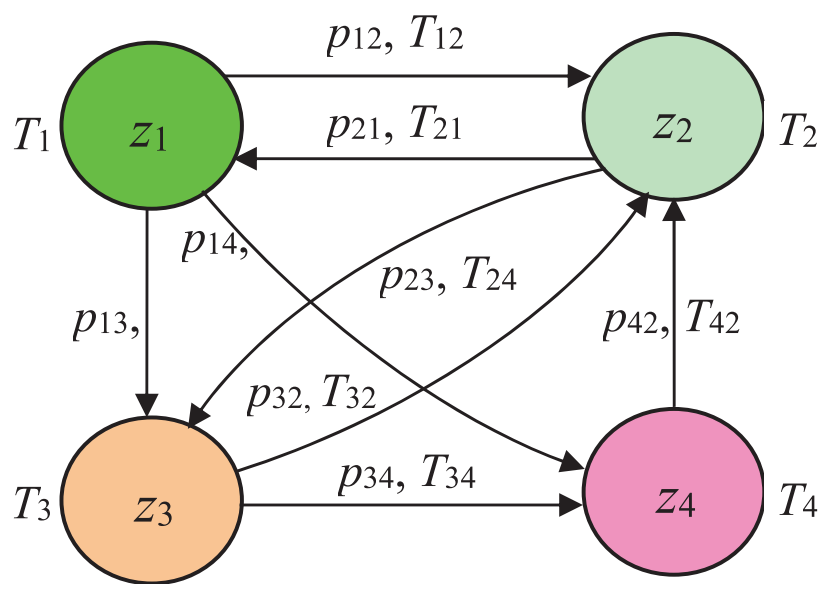

Fig.1. Graph of state transitions, $z i \in Z(i=1,2,3,4)$, for the process $\{Y(t): t \geq 0\}$ of marine power plant device operation: $\left(z_{1}\right)$ - state of active operation of the device with full serviceability, $\left(z_{2}\right)$ - state of passive use of the device with full serviceability, $\left(z_{3}\right)$ - state of planned preventive service of the device with partial serviceability, $\left(z_{2}\right)$ - state of unplanned service of the device (forced by its damage), which is therefore in unserviceability state, $T_{i}$ - time interval of existence of state $z_{i}(i=1,2,3,4), T_{i j}$ - time interval of existence of state zi provided that the next state is $z_{j}(i, j=1,2,3,4 ; i \neq j), p_{i j}$ - probability of state transition from $z_{i}$ to $z_{j}$ in process $\{Y(t): t \geq 0\}$

Arcs in the graph shown in Fig. 1 result from the need to ensure rational operation of a device installed in an arbitrary marine power plant $[2,9,10,11,12]$.

For the device user, the most important state is $z_{1}$, which exists when the device performs its task. Once the task is completed, the state $z_{1}$ changes to $z_{2}$, which occurs with probability $p_{12}$ after time $T_{12}$. When the device is still in the technical state $s_{1}$, which makes it possible to perform the next task, the user of this device may initiate this task, which will result in device's state transition from $z_{2}$ again to $z_{1}$. This transition takes place with probability $p_{21}$ after time $T_{21}$. When the user, based on diagnostic tests, concludes that the device is in technical state which precludes performing the next task, he makes a decision that the device should get preventive 
service. This is equivalent with state transition from $z_{2}$ to $z_{3}$, taking place with probability $p_{23}$ after time $T_{23}$. Once the service is completed, the state $z_{3}$ changes again to $z_{2}$, which takes place with probability $p_{32}$ after time $T_{32}$. The need for preventive service can be observed by the user when the device performs its task. Then, immediately after task completion, the user initiates device's state transition from $z_{1}$ to $z_{3}$, which takes place with probability $p_{13}$ after time $T_{13}$. After completing the preventive service, the device changes state from $z_{3}$ again to $z_{2}$, which takes place with probability $p_{32}$ after time $T_{32}$. However, it is possible that the device gets damaged when performing its task. Then it should get unplanned service, forced by the damage, which changes its state from $z_{1}$ to $z_{4}$. This transition takes place with probability $p_{14}$ after time $T_{14}$. After completing the unplanned service, the device's state transition from $z_{4}$ to $z_{2}$ takes place with probability $p_{42}$ after time $T_{42}$. In rational operation of the device, there is also the relation between states $z 3$ and $z 4$ which describes the situation in state $z_{3}$, being the result of preventive service execution, during which it may turn out that the device got damaged when in state $z_{1}$ and this was not detected by the diagnosing system $(S D G)$ being unable to detect such damage types. In that case, after completing the preventive service, the unplanned service forced by the damage is done, which changes the device's state from $z_{3}$ to $z_{4}$. This state transition takes place with probability $p_{34}$ after time $T_{34}$. Certainly, each marine power plant device can change state to $z_{2}$ from both, states $z_{3}$ and $z_{4}$ (Fig. 1).

The above-described operational situation is illustrated in Fig. 2 as the realisation $y(t)$ of the process $\{Y(t): t \geq 0\}$ of transitions of operation process states $z_{i}(i=1,2,3,4)$ for an arbitrary device during its inter-overhaul period.

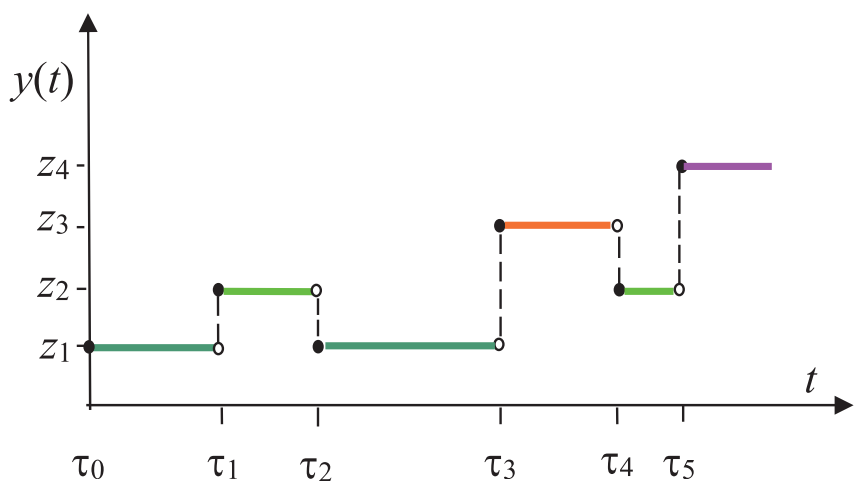

Fig. 2. Sample realisation of process $\{Y(t): t \geq 0\}$ for an arbitrary marine power plant device: $t$-operating time, $\left(z_{1}\right)$ - state of active operation of the device with full serviceability, $\left(z_{2}\right)$ - state of passive use of the device with full serviceability, $\left(z_{3}\right)$ - state of planned preventive service of the device with partial serviceability, $\left(z_{4}\right)$ - state of unplanned service (forced by damage) of the device being in unserviceability state

The interpretation of operation process states $z_{i} \in Z(3)$ can be more precise for a given marine power plant device. For the ship main engine $(S G)$, for instance, the interpretation of these states $z_{i} \in Z(i=1,2,3,4)$ is as follows:

- $\quad$ state $z_{1}=\left(e_{1}, s_{1}\right)$, which has place when the main engine is simultaneously in full serviceability state $\left(s_{1}\right)$ and active operation state $\left(e_{1}\right)$. In this operation process state, the main engine works and generates the average torque $M_{o}$ at rotational speed $n$ at which the overall efficiency of the propulsion is $\eta_{o} \neq \eta_{o}(\max )$, or generates the average torque $M_{o}$ at rotational speed $n$ to ensure the optimal overall propulsion efficiency $\eta_{o}=\eta_{o}(\max )$, etc.,

- $\quad$ state $z_{2}=\left(e_{2}, s_{1}\right)$, which has place when the main engine is simultaneously in passive operational state $\left(e_{2}\right)$ and full serviceability state $\left(s_{1}\right)$. In this operation process state, the main engine is stopped at engine room temperature $t \leq 0{ }^{\circ} \mathrm{C}$ or at temperature $t_{s}>0{ }^{\circ} \mathrm{C}$, etc., and waits for start,

- $\quad$ state $z_{3}=\left(e_{3}, s_{2}\right)$, which has place when the main engine is simultaneously in state of preventive service $\left(e_{3}\right)$ and in partial serviceability state $\left(s_{2}\right)$. This operation process state can result, for instance, from the need to check the quality of fuel spraying by injectors, with possible correction of injection pressure, or to adjust the fuel injection advance angle, etc.,

- $\quad$ state $z_{4}=\left(e_{4}, s_{3}\right)$, which has place when the main engine is in state of unplanned service $\left(e_{4}\right)$ forced by damage, which means that it is also in unserviceability state $\left(s_{3}\right)$. This operation process state can result, for instance, from the need to replace a damaged injector, or broken piston rings, or injection pump with plunger seized in the cylinder, etc.

The performed operation tests have shown that the created model $\{Y(t): t \geq 0\}$ of a real operation process, with the values (states) $z_{i} \in Z(i=1,2,3,4)$ that describe simultaneous existence of mutually implicating technical states $s_{l}(l=1$, $2,3)$ and operational states $e_{k}(k=1,2,3,4)$ taking place in the operational phase of a marine power plant device, is the semi-Markov model, as it is characterised by the following properties $[7,9,11,13,14,19,24]$ :

1) the Markov condition for the evolution of future state transitions of the operation process $\{Y(t): t \geq 0\}$ of each marine power plant device to depend only on the state of this device at a given time and not on its functioning in the past is met; in other words: the future of the device depends only on its present, and not on its past;

2) random variables $T_{i}$ (which are time intervals of existence of state $z_{i}$ regardless of which state appears next) and $T_{i j}$ (which are time intervals of existence of state $z_{i}$ provided that the next process state is $z_{j}$ ) have distributions different than the exponential distribution.

The properties 1 and 2, which justify considering the model $\{Y(t): t \geq 0\}$ of the real operation process $\left\{Y^{\star}(t): t \geq 0\right\}$ as the semi-Markov process, are, respectively, the consequences $K_{1}$ and $K_{2}$ of the following hypothesis: the semi-Markov process $\{Y(t): t \geq 0\}$ can be the model of a real operation process of an arbitrary marine power plant device, as the arbitrary state $z_{i}(i=1,2,3,4)$ of this device and the time interval of its existence depend on the state $z_{j}(j=1,2,3,4 ; j \neq i)$ directly preceding it, and not on earlier states and their time intervals.

Verifying the hypothesis $H$ requires recognising the 
correctness of the following syntactic implication [7, 10, 20, 22]:

$$
H \Rightarrow K_{i}(i=1,2)
$$

Then the non-deductive (inductive) inference conducted in accordance with the following scheme can be applied [10, 21, 22]:

$$
\left[K_{i}(i=1,2), H \Rightarrow K_{i}(i=1,2] \vdash H\right.
$$

Logical interpretation of this inference scheme is as follows: if experimental verification of consequences $K_{i}(i=1,2)$ has confirmed their truthfulness, then if the implication (4) is true then the hypothesis $H$ is also true and can be accepted. The inductive inference conducted in accordance with the above scheme (5) bears the name of reductive inference. Certainly, like each other inference belonging to the group of inductive inferences, this inference leads only to possible, and not sure conclusions $[7,20,22]$.

The property 1 is met (and hence the consequence $K_{1}$ true), as contemporary marine power plant devices are treated as diagnosed systems $(S D N)$ which are to be equipped with advanced computer diagnosing systems $(S D G)$. This makes a basis for formulating complete and highly reliable diagnoses (temporary diagnosis, genesis, and prognosis) concerning the technical condition of the device at arbitrary time $t$ of its operation $[4,5,10,11,12,17,18]$. Consequently, the $S D N$ user gets satisfactory insight into the current technical condition of each device, which allows him to make a right decision whether state $z_{3}$ or state $z_{4}$ should be introduced, as these two states cannot exist simultaneously. Therefore, if the process $\{Y(t): t \geq 0\}$ stays in one of these states, $\left(z_{3}\right.$ or $\left.z_{4}\right)$, then its future state $z_{2}$ and time interval $T_{2}$ of its existence (Fig. 1) will depend only on the state $z_{3}$ or $z_{4}$ directly preceding it. That means that state $z_{2}$ and its time interval $T_{2}$ do not depend on state $z_{1}$ which existed before state $z_{3}$ or $z_{4}$. But if the process $\{Y(t): t \geq 0\}$ stays first in state $z_{1}$, and then changes to state $\mathrm{z}_{2}$, then the state $z_{2}$ and its time interval $T_{2}$ will depend only on state $z_{1}$, and not additionally on one of earlier states $z_{3}$ or $z_{4}$. Also, the state $z_{1}$ and its time interval $T_{1}$ will only depend on state $z_{2}$ directly preceding it, and not on earlier states $z_{3}$ or $z_{4}$ (Fig. 1).

The property 2 is also met (and hence the consequence $K_{2}$ true), as marine power plant devices, in particular mechanical devices, suffer damages resulting from excessive surface and/or volumetric wear caused by the action of so-called cumulative stimuli, more rarely relaxation stimuli $[3,5,6,11$, 12]. These devices are not subject to the action of so-called stepwise stimuli, as there are not impact loads which could damage them regardless of their technical condition. Therefore, the exponential distribution cannot be used for probabilistic description of random variables $T_{1}, T_{12}, T_{13}$, and $T_{14}$. The use of this distribution would only be justified when:

- the level of strength properties (wear resistance) of the devices did not change, which means no ageing provoked damages (coming from cumulative inputs),
- device damages were caused by accidental external or internal impact actions, so-called stepwise stimuli, either being the consequence of device operation or not.

The distributions of the remaining random variables also cannot be considered exponential distributions. This results not only from technical conditions, but also from organisational and economic conditions of the existence of states $z_{1}, z_{2}, z_{3}$, and $z_{4}[5,6,11,12]$.

Hence, we can conclude that the developed model $\{Y(t): t \geq 0\}$ of the real operation process $\left\{Y^{\star}(t): t \geq 0\right\}$, which is characterised by transitions of states $z_{i}(i=1,2,3,4)$ to states $z_{j}(i, j=1,2,3,4 ; j \neq i)$ taking place during the operation of marine power plant devices, is the semi-Markov model. This justifies the application of the theory of semi-Markov processes to determine reliability characteristics of these devices, while the application of decision-making (control) theories to the semi-Markov processes enable making optimal operating decisions, with profit used as the optimisation criterion. The semi-Markov models of real processes are characterised by the initial distribution and the functional matrix bearing the name of the kernel of the process $[13,14]$.

The initial distribution of the process $\{Y(t): t \geq 0\}$ (Fig. 2 ) is given by the formula:

$$
P_{i}=P\left\{Y(0)=z_{i}\right\}=\left\{\begin{array}{l}
1 \text { for } i=1 \\
0 \text { for } i=2,3,4
\end{array}\right.
$$

while its functional matrix (kernel), according to the graph shown in Fig. 1, has the following form:

$$
\mathbf{Q}(\mathbf{t})=\left[\begin{array}{cccc}
0 & Q_{12}(t) & Q_{13}(t) & Q_{14}(t) \\
Q_{21}(t) & 0 & Q_{23}(t) & 0 \\
0 & Q_{32}(t) & 0 & Q_{34}(t) \\
0 & Q_{42}(t) & 0 & 0
\end{array}\right]
$$

where:

$Q_{i j}(t)=p_{j} F_{j}(t)$ is the kernel element of the process (7), which is the conditional probability of appearance of state $z_{j}$ at time not longer than $t$, provided that the previous process state was $z_{i}(i, j=1,2,3,4 ; j \neq i)$,

$p_{i j}$ - probability of transition of process $\{Y(t): t \geq 0\}$ from state $z_{i}$ to $z_{j}$,

$F_{i j}(t)$ - cumulative distribution function of random variable $T$,

Methods to determine the probability $p_{i j}$ and the cumulative distribution function $F_{i j}(t)$ are well known and have been presented in numerous publications, for instance in monographs $[12,13]$.

It results from the presented hypothesis that the model $\{Y(t): t \geq 0\}$ of real operation processes of marine power plant devices is the semi-Markov process, as it is characterised by the fact that the time interval of the process state existing at time $\tau_{\mathrm{n}}$ and the process state which can appear at time $\tau_{\mathrm{n}+1}$ do not depend stochastically on earlier states and their time intervals. In marine power plant device operation (Fig. 2), it is important for the realisation $y(t)$ of the process $\{Y(t): t \geq 0\}$ to be optimal. This can be achieved by applying the decisionmaking (control) theory of semi-Markov processes to the 
analysed process $\{Y(t): t \geq 0\}$ considered as the semi-Markov decision-making process $[6,9,12,15,16,21]$.

The semi-Markov decision-making process $\{\mathrm{Y}(\mathrm{t}): \mathrm{t} \geq 0\}$ is the process, the realisation of which depends on decisions $d_{i}(\tau)(\mathrm{i}=1,2,3,4)$, made at times $\tau$ : first at initial time $\tau_{0}$, and then at state transition times $, \tau_{1}, \ldots, \tau_{n}, \ldots$ of this process. Decision $d_{i}\left(\tau_{n}\right)$ is the decision made at time, when the semiMarkov process is in the operation state zi, which means that $Y\left(\tau_{n}\right)=z_{i}(i=1,2,3,4)$. In each state of process $\{\mathrm{Y}(\mathrm{t}): \mathrm{t} \geq 0\}$, the decision $d_{i}$ belonging to the set of decisions, $D_{i}\left(d_{i} \in D_{i}\right)$, can be made. Making decision $d_{i}$ means selecting $i$-th row of the functional matrix (kernel) expressed by the relation (8) which results from matrix (7).

$$
\mathbf{Q}(\mathbf{t})=\left[\begin{array}{cccc}
0 & Q_{12}^{d_{1}}(t) & Q_{13}^{d_{1}}(t) & Q_{14}^{d_{1}}(t) \\
Q_{21}^{d_{2}}(t) & 0 & Q_{23}^{d_{2}}(t) & 0 \\
0 & Q_{32}^{d_{3}}(t) & 0 & Q_{34}^{d_{3}}(t) \\
0 & Q_{42}^{d_{4}}(t) & 0 & 0
\end{array}\right]
$$

where:

$Q_{i j}^{d_{k}}(t)=p_{i j}^{d_{k}} F_{i j}^{d_{k}}(t)$ is the kernel element of the process (8), which is the conditional probability of appearance of state $z_{j}$ at time not longer than $\mathrm{t}$ when the decision $d_{k}$ is made, provided that the previous process state was $z_{i}$,

$p_{i j}^{d_{k}}$ - probability of transition of process $\{Y(t): t \geq 0\}$ from state zi to zj when the decision $\mathrm{dk}$ is made,

$F_{i j}^{d_{k}}(t)$ - cumulative distribution function of random variable $T_{i j}^{d_{k}}$,

The probabilities $p_{i j}^{d_{k}}$ and cumulative distribution functions $F_{i j}^{d_{k}}(t)$ are determined in a similar way as for the earlier mentioned probabilities $p_{i j}$ and cumulative distribution functions $F_{i j}(t)$ characterising the functional matrix (7).

The i-th row of the functional matrix (8) defines the probabilistic mechanism of process evolution in time interval $\left[\tau_{n}, \tau_{n+1}\right)$. This row is selected from the set:

$$
\left\{Q_{i j}^{\left(d_{i}\right)}(t): t \geq 0, d_{i} \in D_{i} ; i, j=1,2,3,4, i \neq j\right\},
$$

Decision $d_{i}\left(\tau_{n}\right)=z_{i} \in D_{i}$ means that the semi-Markov process evolves in such a way that, according to the distribution $\left(p_{i l}^{\left(d_{i}\right)}: l=1,2,3,4\right)$, the process state $z_{j}$ is selected to which the process changes at time $\tau_{n+1}$. The length of the time interval $\left[\tau_{n}, \tau_{n+1}\right)$ is determined in accordance with the distribution given by the cumulative distribution function $F_{i j}^{\left.d_{i} k\right)}(t)$. This results from the fact that $Q_{i j}^{\left(d_{i}\right)}(t)=p_{i j}^{\left(d_{i}\right)} F_{i j}^{(d)}[12,13,15,21]$.

Decisions are made in accordance with the adopted strategy

$$
d=\left\{\left(d_{1}\left(\tau_{1}\right), \ldots, d_{N}\left(\tau_{n}\right)\right): n=0,1,2, \ldots\right\}
$$

comprising possible decisions $d_{i} \in D_{i}$ which may be made. The elements of the decision sequence (10) are vectors with components being the decisions made in given states, at times of their transitions.

In the case of marine power plant devices, the strategy $d$ is the markovian strategy, as for each state $z_{i} \in Z(3)$ and each state transition time $\tau_{n}, n=1,2, \ldots$, the decision $d_{i}\left(\tau_{n}\right) \in D_{i}$ does not depend on process realisation until time $\tau_{n}$. Moreover, this decision does not depend on $n$, and $d_{i}\left(\tau_{n}\right)=d_{i}$, which means that it is also the stationary strategy, and the semiMarkov decision-making process $\{Y(t): t \geq 0\}$ is the uniform process. With respect to marine power plant devices, the optimisation of the semi-Markov decision-making process $\{Y(t): t \geq 0\}$ consists in selecting a strategy for which profit, being the optimisation criterion, takes the maximum value.

\section{OPTIMISING THE SEMI-MARKOV DECISION-MAKING PROCESS AS THE FOUR-STATE MODEL OF MARINE POWER PLANT DEVICE OPERATION PROCESS}

Optimisation of the semi-Markov process $\{Y(t): t \geq 0\}$ with functional matrix (8) and initial distribution (6), being the model of marine power plant device (USO) operation process, is always done for a given time interval $\left[0, \tau_{m}\right)$ of device operation. That means that the number of transitions of states $z_{i} \in Z$ (4) of this process is predetermined.

The optimisation criterion is the profit gained in time unit of existence of state $\mathrm{zi}$, which is given by relation [12, $13,16,21]$ :

$$
r_{i j}^{\left(d_{k}\right)}(t), \quad i, j=1,2,3,4, d_{k} \in D_{i}
$$

provided that at the transition time this state changes to $z_{j}$, and decision $d_{k} \in D_{i}$ was made at the time of state transition to $z_{i}$. The unit profit $r_{i j}^{d_{k}}(t)$ is the function of time t calculated from the time of appearance of state $z_{i}$ until the time of appearance of state $z_{j}$.

When the marine power plant device (USO) is in state, the transport task is executed by the ship. Once this task is completed, the crew is paid the salary, which means that they have the unit profit $r_{1 j}^{\left(d_{k}\right)}(t), j=2,3,4$, regardless of whether the USO changes next to state $z_{2}, z_{3}$, or $z_{4}$. This also means that in the mathematical sense, $r_{12}^{d_{k}}>0, r_{13}^{d_{k}}>0, r_{14}^{d_{k}}>0$. On the other hand, the existence of states $z_{2}, z_{3}$, and $z_{4}$ generates costs, which in the mathematical sense can be considered negative profits. The cost $r_{21}^{d_{k}}<0$ (as negative profit) of USO state transition from $z_{2}$ to $z_{1}$ results from the need to spend financial resources to ensure that USO will return to the active operation state $\left(z_{1}\right)$. For instance, returning the main engine $(S G)$ to state $z_{1}$ requires purchasing energy media, such as fuel (heavy and/or diesel oil), lubricating oil, technical water for engine cooling, etc. Also, state transition from $z_{2}$ to $z_{3}$ of either $S G$ or any other USO involves costs related with crew activity to prepare conditions for preventive service $\left(z_{3}\right)$, hence the (negative) profit $r_{23}^{d_{k}}<0$ is to be considered. In turn, the execution of preventive service to obtain state transition from $z_{3}$ to $z_{2}$ requires incurring the cost $r_{32}^{d_{k}}<0$. Likewise, the unplanned service forced by its damage which is necessary to bring the device from state $z_{4}$ to $z_{2}$ requires incurring the cost $r_{42}^{d_{k}}<0$. Also, when a damage to $S G$ or another USO is detected when performing the planned service, the cost $r_{34}^{d_{k}}<0$ is to be incurred to change the state from $z_{3}$ to $z_{4}$. The 
state $z 4$ is related with the unplanned service, forced by the damage and performed to bring the device back to state $z 2$. This state transition involves the cost $r_{42}^{d_{k}}<0$.

Taking into account the above characterised unit profits $r_{i j}^{d_{k}}(t)$, the total profit obtained until time $t$, as calculated from the time of appearance of state $z_{i}$ of the process $\{Y(t): t \geq 0\}$ but before transition to state $z_{j}$, is given by the function $[12,13]$ :

$$
R_{i j}^{\left(d_{k}\right)}(t)=\int_{0}^{t} r_{i j}^{\left(d_{k}\right)}(\tau) d \tau, \quad i, j=1,2,3,4, d_{k} \in D_{i}
$$

In the economic sense, the profit is gained when the state $z_{1}$ exists, while all remaining states $z_{i}(i=2,3,4)$, generate costs (losses) which, as already mentioned, can be considered in the mathematical approach as negative profits (with a minus sign). Except for the main engine ( $S G)$, this fact is not always taken into consideration, which results from the fact that the ship with non-functioning engine cannot perform transport tasks. Moreover, when the ship is at sea in rough weather conditions, the main engine failure can lead to the loss of the ship with all transported cargo, which is equivalent for the ship owner to substantial financial loss.

Along with the profit defined by relation (12), another factor which needs considering is a one-off cash inflow (profit) which gives $b_{\ddot{j}}^{d_{k}}>0$, or a one-off cost, with $b_{\ddot{j}}^{d_{k}}<0$. The constant one-off profit and constant one-off cost (negative profit) resulting from state transition from $z_{i}$ to $z_{j}(i, j=1,2$, $3,4)$ are given by the formula $[12,13]$ :

$$
b_{i j}^{\left(d_{k}\right)}, \quad i, j=1,2,3,4, i \neq j, d_{k} \in D_{i}
$$

In the model $\{Y(t): t \geq 0\}$, the cash inflows, i.e. profits $b_{12}^{d_{k}}>0, b_{13}^{d_{k}}>0$ and $b_{14}^{d_{k}}>0$, are to be paid by the ship owner to the crew to ensure start-up of the works related with the appearance of states $z_{2}, z_{3}$ and $z_{4}$. For instance, when the $S G$ stays in state $z_{2}$, the profit $b_{12}^{d_{k}}>0$, as the crew has to get financial resources to purchase fuel, lubricating oil, spare parts, etc. When in future, the ship owner is commissioned to perform the transport task, these purchases will provide the opportunity for $S G$ state transition from state $z_{2}$ to $z_{1}$ and starting the execution of the commissioned task. Also, rational state transition of either $S G$, or another USO from state $z_{1}$ do $z_{3}$ requires involving financial resources to purchase spare parts for the preventive service to be started. For the $S G$ for instance, this requires purchasing: injectors, filter cartridges, injector pumps, etc., hence $b_{13}^{a_{k}}>0$. Likewise, the crew gets financial resources for necessary purchases to ensure rational state transition of either $S G$ o any other USO from state $z_{1}$ to $z_{4}$, hence also $b_{14}^{d_{k}}>0$. In turn, state transition from $z_{2}$ to $z_{1}$ is related with the costs incurred by the crew to purchase fuel and lubricating oil, as well as crew salaries to be paid to maintain the existence of state $z_{1}$. Therefore, state transition from $z_{2}$ to $z_{1}$ requires incurring oneoff cost, hence $b_{21}^{d_{k}}<0$. Moreover, the cost $b_{23}^{d_{k}}<0$ should be determined which allows state transition from $z_{2}$ to $z_{3}$, and the cost $b_{32}^{d_{k}}<0$ for the transition from state $z_{3}$ to $z_{2}$, as well as the cost $b_{34}^{d_{k}}<0$ for the state transition from $z_{3}$ to $z_{4}$.

The total profit taking into account relations (12) and (13) is given by the function $[12,13]$ :

$$
U_{i}^{\left(d_{k}\right)}=\sum_{j \in S} \int_{0}^{\infty}\left(R_{i j}^{\left(d_{k}\right)}(t)+b_{i j}^{\left(d_{k}\right)}\right) d Q_{i j}^{\left(d_{k}\right)}(t),
$$

representing the expected profit to be gained in a single interval of realisation of state $z_{i}$, when the decision $d_{k} \in D_{i}$ was made at the beginning of this state.

Analysing profits gained from the realisation of the operation process in time interval $\left[0, \tau_{m}\right)$ requires defining the expected (average) profit $V_{i}\left(d_{m}\right), z_{i} \in Z, i=1,2,3,4$ to be gained as a result of the adopted decision-making strategy $d_{m}(15)$.

In the USO operation phase, making a decision (choosing from the set of possible decisions) at time $\tau_{n}$ does not depend on the past realisation of the operation process, but on the technical condition of the device. That means that the Markov strategy can be applied $[12,13]$

$$
d_{m}=\left\{\left(d_{1}\left(\tau_{n}\right), \ldots, d_{N}\left(\tau_{n}\right)\right): n=0,1, \ldots, m-1\right\}
$$

provided that the initial state of the process is state $z_{i} \in Z$.

Then we can determine the expected (average) profit $V_{i}\left(d_{m}\right), z_{i} \in Z, i=1,2,3,4$ to be gained from time $\tau_{1} \in\left[0, \tau_{m}\right)$, i.e. in time interval $\left[\tau_{1}, \tau_{m}\right)$ when applying the strategy

$$
d_{m-1}=\left\{\left(d_{1}\left(\tau_{n}\right), \ldots, d_{N}\left(\tau_{n}\right)\right): n=1, \ldots, m-1\right\},
$$

provided that state $z_{j} \in Z$ was initiated at time $\tau_{1}$.

Thus, the expected profit to be obtained as a result of realisation of the USO operation process in time interval $\left[\tau_{0}, \tau_{m}\right)$ is the sum of the expected profit gained in time interval $\left[\tau_{0}, \tau_{1}\right)$ and the profit expected to be gained in time interval $\left[\tau_{1}, \tau_{m}\right)$, which can be expressed by the formula:

$$
V_{z_{i}}\left(d_{m}\right)=U_{z_{i}}^{\left(d_{k}\right)}+\sum_{z_{j} \in Z} p_{i j}^{\left(d_{k}\right)} V_{z_{j}}\left(d_{m-1}\right), z_{i} \in Z \text {. }
$$

Taking into consideration formula (14), we get the function:

$$
\begin{aligned}
V_{z_{i}}\left(d_{m}\right)= & \sum_{z_{j} \in Z} \int_{0}^{\infty}\left(R_{i j}^{\left(d_{k}\right)}(t)+b_{i j}^{\left(d_{k}\right)}\right) d Q_{i j}^{\left(d_{k}\right)}(t)+ \\
& \sum_{z_{j} \in Z} p_{i j}^{\left(d_{k}\right)} V_{z_{j}}\left(d_{m-1}\right), z_{i} \in Z .
\end{aligned}
$$

In the USO operation phase, of high importance are optimal strategies, as they provide opportunities for gaining maximum profits. Strategy $d_{m}^{*}$ can be considered the optimal strategy when it allows the maximum profit to be gained

$$
V_{z_{i}}\left(d_{m}^{*}\right)=\max _{d_{m}}\left[V_{z_{i}}\left(d_{m}\right)\right]
$$

in time interval $\left[\tau_{0}, \tau_{m}\right)$ for the semi-Markov decisionmaking process starting from state $e_{i}$.

That means that the inequality [13]

$$
V_{z_{i}}\left(d_{m}^{*}\right) \geq V_{z_{i}}\left(d_{m}\right)
$$

is met for all possible strategies $d_{m}$. 
The optimal strategy can be found using the dynamic programming method, based on a well-known Bellman's principle of optimality. In the analysed case, this principle can be formulated as follows $[1,13]$ :

When $z_{j} \in Z$ is the state of process $\{Y(t): t \geq 0\}$ existing at time $\tau_{1}$ as a result of a decision made at time $\tau_{0}=0$ then, if the strategy

$$
d_{m}^{*}=\left\{\left(d_{1}^{*}\left(\tau_{n}\right), \ldots, d_{N}^{*}\left(\tau_{n}\right)\right): n=0,1, \ldots, m-1\right\}
$$

is the optimal strategy, then the strategy

$$
d_{m-1}^{*}=\left\{\left(d_{1}^{*}\left(\tau_{n}\right), \ldots, d_{N}^{*}\left(\tau_{n}\right)\right): n=1, \ldots, m-1\right\}
$$

is also the optimal strategy for the process initiated from state $z_{j}$ at time $\tau_{1}$.

Based on this principle and formula (17), the expected (average) profit is given as:

$$
V_{z_{i}}\left(d_{m}^{*}\right)=\max _{d_{k} \in D_{i}}\left[U_{i}^{\left(d_{k}\right)}+\sum_{z_{j} \in Z} p_{i j}^{\left(d_{k}\right)} V_{z_{j}}\left(d_{m-1}^{*}\right)\right], z_{i} \in Z
$$

When at time $\tau_{m-1}$, the operation process is in state $z_{i} \in Z$, then the optimal strategy is determined from the relation

$$
V_{z_{i}}\left(d_{1}^{*}\right)=\max _{d_{k} \in D_{i}}\left[U_{i}^{\left(d_{k}\right)}\right], z_{i} \in Z
$$

Consequently, the optimal strategy is given as:

$$
d_{1}^{*}=\left\{\left(d_{1}^{*}\left(\tau_{n}\right), \ldots, d_{N}^{*}\left(\tau_{n}\right)\right): n=m-1\right\}
$$

After calculating the maximum profit $V_{i}\left(d_{1}^{*}\right)=\max _{d_{k} \in D_{i}}\left[U_{i}^{d_{k}}\right]$ for each $z_{i} \in Z(i=1,2,3,4)$, the optimal strategy

$$
d_{2}^{*}=\left\{\left(d_{1}^{*}\left(\tau_{n}\right), \ldots, d_{N}^{*}\left(\tau_{n}\right)\right): n=m-2, m-1\right\}
$$

is determined for the operation process $\{Y(t): t \geq 0\}$, the initial time of which is $\tau_{m-2}$. This strategy is determined from the relation

$$
V_{z_{i}}\left(d_{2}^{*}\right)=\max _{d_{k} \in D_{i}}\left[U_{i}^{\left(d_{k}\right)}+\sum_{z_{j} \in Z} p_{i j}^{\left(d_{k}\right)} V_{z_{j}}\left(d_{1}^{*}\right)\right], z_{i} \in Z
$$

Continuing this procedure, we obtain the optimal decisionmaking strategy

$$
d_{m}^{*}=\left\{\left(d_{1}^{*}\left(\tau_{n}\right), \ldots, d_{N}^{*}\left(\tau_{n}\right)\right): n=0,1, \ldots, m-2, m-1\right\}
$$

The optimal strategy of operating decision-making (26) makes it possible to determine the next state $z_{j}$, when the process is in state $z_{i}(i, j=1,2,3,4 ; i \neq j)$, in such a way that the user of an arbitrary USO can gain the maximum profit. Applying the above procedure to determine the optimal strategy of operating decision-making requires taking into consideration the initial distribution of the model $\{Y(t): t \geq 0\}$ of the operation process, with the initial distribution given by formula (6) and the functional matrix (kernel) in the form (8). Certainly, matrix (8) is the matrix (7) modified such that its functions $Q_{i j}(t)$ depend on decisions $d_{k}$.

Past examinations of USO operations have shown that the time intervals of existence of states $z_{i} \in Z, i=1,2,3,4$ and the times of state transition from $z_{i}$ to $z_{j}$ of the process $\{Y(t): t \geq 0\}$ can be considered random variables with gammadistribution $[10,11,12]$. Hence, the elements of matrix (8)

$$
\left\{Q_{i j}^{\left(d_{k}\right)}(t): t \geq 0, d_{k} \in D_{i}, i, j \in\{1,2,3,4\}, i \neq j\right\}
$$

can be expressed as

$$
Q_{i j}^{\left(d_{k}\right)}(t)=\int_{0}^{t} q_{i j}^{\left(d_{k}\right)}(\tau) d \tau,
$$

where:

$$
q_{i j}^{\left(d_{k}\right)}(\tau)=p_{i j}^{d_{k}} \frac{\lambda_{i j}^{\left(d_{k}\right) \alpha_{i j}^{\left(d_{k}\right)}}}{\Gamma\left(\alpha_{i j}^{\left(d_{k}\right)}\right)} \tau^{\alpha_{i j}^{\left(d_{k}\right)}-1} e^{-\lambda_{i j}^{\left(d_{k}\right)} \tau}
$$

The function

$$
f_{i j}^{\left(d_{k}\right)}(\tau)=\frac{\lambda_{i j}^{\left(d_{k}\right) \alpha_{i j}^{\left(d_{k}\right)}}}{\Gamma\left(\alpha_{i j}^{\left(d_{k}\right)}\right)} \tau^{\alpha_{i j}^{\left(d_{k}\right)}-1} e^{-\lambda_{i j}^{\left(d_{k}\right)} \tau}
$$

is the gamma distribution density. In this distribution, the expected value $m_{i j}^{d_{k}}$ and the standard deviation $\sigma_{i j}^{d_{k}}$ are given by formulas:

$$
m_{i j}^{\left(d_{k}\right)}=\frac{\alpha_{i j}^{\left(d_{k}\right)}}{\lambda_{i j}^{\left(d_{k}\right)}}, \quad \sigma_{i j}^{\left(d_{k}\right)}=\frac{\sqrt{\alpha_{i j}^{\left(d_{k}\right)}}}{\lambda_{i j}^{\left(d_{k}\right)}}
$$

Hence, the shape parameters $\alpha_{i j}^{\left(d_{k}\right)}$ and the scale parameters $\lambda_{i j}^{\left(d_{k}\right)}$ of this distribution can be expressed as:

$$
\alpha_{i j}^{\left(d_{k}\right)}=\frac{\left[m_{i j}^{\left(d_{k}\right)}\right]^{2}}{\left[\sigma_{i j}^{\left(d_{k}\right)}\right]^{2}}, \quad \lambda_{i j}^{\left(d_{k}\right)}=\frac{m_{i j}^{\left(d_{k}\right)}}{\left[\sigma_{i j}^{\left(d_{k}\right)}\right]^{2}}
$$

Applying the above procedure of making optimal decisions, i.e. decisions which will give the USO user the opportunity to gain the maximum profit, requires defining the most profitable state. In the analysed case, this state is $z_{1}$, in which the engine moves the ship, thus enabling the performance of the transport task and providing profit to the ship owner. According to the graph in Fig. 1 and the functional matrix (8), the user of an arbitrary USO being in state $z_{1}$ can make one of three possible decisions: $d_{1}$ - change to state $z_{2}, d_{2}-$ change to state $z_{3}$, and $d_{3}$ - change to state $z_{4}$. Therefore, the set of decisions for state $z_{1}$ is as follows:

$$
D_{1}=\left\{d_{1}, d_{2}, d_{3}\right\}
$$

Each decision $d_{k} \in D_{1}, k=1,2,3$ (33), is attributed with time interval of operation state $\left(z_{1}\right)$, probability of state transition to $z_{2}, z_{3}$ and $z_{4}$, and profit gained from task realisation.

It results from the proposed four-state model of operation process of an arbitrary USO with states $z_{i} \in Z(i=1,2,3$, $4)$, matrix (8) and the graph shown in Fig. 1 that making a decision requires determining the following operating parameters:

1. Probabilities $p_{i j}^{d_{k}}$ of state transitions, i.e.:

$p_{12}^{(1)}, p_{13}^{(1)}, p_{14}^{(1)}, p_{12}^{(2)}, p_{13}^{(2)}, p_{14}^{(2)}, p_{12}^{(3)}, p_{13}^{(3)}, p_{14}^{(3)}$, 
2. Conditional expected values $m_{i j}^{d_{k}}$ (of operating state time intervals $\left.T_{i j}^{d_{k}}\right)$, i.e.: $m_{12}^{(1)}, m_{13}^{(1)}, m_{14}^{(1)}, m_{12}^{(2)}, m_{13}^{(2)}, m_{14}^{(2)}, m_{12}^{(3)}, m_{13}^{(3)}, m_{14}^{(3)}$,

3. Standard deviations $\sigma_{i j}^{d_{k}}$ of conditional state time intervals, i.e.:

$\sigma_{12}^{(1)}, \sigma_{13}^{(1)}, \sigma_{14}^{(1)}, \sigma_{12}^{(2)}, \sigma_{13}^{(2)}, \sigma_{14}^{(2)}, \sigma_{12}^{(3)}, \sigma_{13}^{(3)}, \sigma_{14}^{(3)}$, 4. Average profits $r_{i j}^{d_{k}}$ gained in ship operation time unit, i.e.:

$r_{12}^{(1)}, r_{13}^{(1)}, r_{14}^{(1)}, r_{12}^{(2)}, r_{13}^{(2)}, r_{14}^{(2)}, r_{12}^{(3)}, r_{13}^{(3)}, r_{14}^{(3)}$,

5. Constant one-off profits $b_{i j}^{d_{k}}$ obtained from single operating state realisation, i.e.:

$b_{12}^{(1)}, b_{13}^{(1)}, b_{14}^{(1)}, b_{12}^{(2)}, b_{13}^{(2)}, b_{14}^{(2)}, b_{12}^{(3)}, b_{13}^{(3)}, b_{14}^{(3)}$.

Moreover, the distribution parameters of conditional operating state time intervals for different decisions, which are necessary for decision-making, should be calculated from formulas (32). Therefore, we should calculate:

6. Distribution parameters $\alpha_{i j}^{d_{k}}$ and $\lambda_{i j}^{d_{k}}$ of conditional operating state time intervals, i.e.:

$\alpha_{12}^{(1)}, \alpha_{13}^{(1)}, \alpha_{14}^{(1)}, \alpha_{12}^{(2)}, \alpha_{13}^{(2)}, \alpha_{14}^{(2)}, \alpha_{12}^{(3)}, \alpha_{13}^{(3)}, \alpha_{14}^{(3)}$, $\lambda_{12}^{(1)}, \lambda_{13}^{(1)}, \lambda_{14}^{(1)}, \lambda_{12}^{(2)}, \lambda_{13}^{(2)}, \lambda_{14}^{(2)}, \lambda_{13}^{(3)}, \lambda_{13}^{(3)}, \lambda_{14}^{(3)}$.

After completing the task performed by an arbitrary USO, but when it is still in state $z_{1}$, the user can make a decision that the next state will be $z_{2}$. Then, when the USO is already in state $z_{2}$, the user can make, according to the graph in Fig. 1 and the functional matrix (8), one of two possible decisions: $d_{1}$ - change to state $z_{1}, d_{2}$ - change to state $z_{3}$. Therefore, the set of decisions for state $z_{2}$ is:

$$
D_{2}=\left\{d_{1}, d_{2}\right\}
$$

Also in this case, each decision $d_{k} \in D_{2}, k=1,2$ (34) is attributed with time interval of ready-to-operate stop $\left(z_{2}\right)$, probability of transition to states $z_{1}$ and $z_{3}$, and profit related with the existence of this state. Making a proper decision requires determining the following operating parameters:

1. Probabilities $p_{i j}^{d_{k}}$ of state transitions, i.e.: $p_{21}^{(1)},, p_{23}^{(1)}, p_{21}^{(2)}, p_{23}^{(2)}$,

2. Conditional expected values $m_{i j}^{d_{k}}$ (of state time intervals $\left.T_{i j}^{d_{k}}\right)$, i.e.:

$m_{21}^{(1)}, m_{23}^{(1)}, m_{21}^{(2)}, m_{23}^{(2)}$,

3. Standard deviations $\sigma_{i j}^{d_{k}}$ of conditional state time intervals, i.e.:

$\sigma_{21}^{(1)}, \sigma_{23}^{(1)}, \sigma_{21}^{(2)}, \sigma_{23}^{(2)}$,

4. Average "profits" $r_{i j}^{d_{k}}$ gained in time unit of ready-tooperate stop, i.e.:

$r_{21}^{(1)}, r_{13}^{(1)}, r_{21}^{(2)}, r_{13}^{(2)}$,

5. Constant one-off profits $b_{i j}^{d_{k}}$ obtained from single readyto-operation stop state realisation, i.e.: $b_{21}^{(1)}, b_{23}^{(1)}, b_{21}^{(2)}, b_{23}^{(2)}$;

The distribution parameters of conditional ready-tooperate stop state time intervals for different decisions, which are necessary for decision-making, should be calculated from formulas (32). Therefore, we should calculate:

6. Distribution parameters $\alpha_{i j}^{d_{k}}$ and $\lambda_{i j}^{d_{k}}$ of conditional stop time intervals, i.e.:

$\alpha_{21}^{(1)}, \alpha_{23}^{(1)}, \alpha_{21}^{(2)}, \alpha_{23}^{(2)}, \lambda_{21}^{(1)}, \lambda_{23}^{(1)}, \lambda_{21}^{(2)}, \lambda_{23}^{(2)}$.

When the SG or any other USO, and, consequently, the process $\{Y(t): t \geq 0\}$ are in state $z_{3}$, the user can make, according to the matrix (8) and the graph in Fig. 1, only one of two possible decisions: $d_{1}$ - change to state $z_{2}, d_{2}$ - change to state $z_{4}$. Therefore, the set of decisions for state $z_{3}$ (preventive service) is as follows

$$
D_{3}=\left\{d_{1}, d_{2}\right\}
$$

Like in the previous cases, in this case each decision $d_{k} \in D_{3}$, $k=1,2$ (35) is attributed with time interval of preventive service state $\left(z_{3}\right)$, probability of transition to states $z_{2}$ and $z_{4}$, and profit related with the existence of this state. Making a proper decision requires determining the following operating parameters:

1. Probabilities $p_{i j}^{d_{k}}$ of state transitions, i.e.:

$p_{32}^{(1)}, p_{34}^{(1)}, p_{32}^{(2)}, p_{34}^{(2)}$,

2. Conditional expected values $m_{i j}^{d_{k}}$ (of state time intervals $\left.T_{i j}^{d_{k}}\right)$, i.e.:

$m_{32}^{(1)}, m_{34}^{(1)}, m_{32}^{(2)}, m_{34}^{(2)}$,

3. Standard deviations $\sigma_{i j}^{d_{k}}$ of conditional preventive service time intervals, i.e.:

$\sigma_{32}^{(1)}, \sigma_{34}^{(1)}, \sigma_{32}^{(2)}, \sigma_{34}^{(2)}$,

4. Average "profits" $r_{i j}^{d_{k}}$ gained in time unit of preventive service, i.e.:

$r_{32}^{(1)}, r_{34}^{(1)}, r_{32}^{(2)}, r_{34}^{(2)}$

5. Constant (negative) "profits" $b_{i j}^{d_{k}}$ obtained from single realisation of state $e_{3}$, i.e.:

$b_{32}^{(1)}, b_{34}^{(1)}, b_{32}^{(2)}, b_{34}^{(2)}$

The distribution parameters of conditional preventive service state time intervals for different decisions, which are necessary for decision-making, should be calculated from formulas (32). Therefore, we should calculate:

6. Distribution parameters $\alpha_{i j}^{d_{k}}$ and $\lambda_{i j}^{d_{k}}$ of conditional preventive service time intervals, i.e.:

$\alpha_{32}^{(1)}, \alpha_{34}^{(1)}, \alpha_{32}^{(2)}, \alpha_{34}^{(2)}, \lambda_{32}^{(1)}, \lambda_{34}^{(1)}, \lambda_{32}^{(2)}, \lambda_{34}^{(2)}$.

In the case when the $S G$ or any other USO and, consequently, the process $\{Y(t): t \geq 0\}$ are in state $e_{4}$, the user can make, according to matrix (8) and the graph in Fig. 1, only one decision: $d_{1}$ - change to state $z_{2}$. Therefore, the set of decisions for state $z_{4}$ (unplanned service forced by damage) has only one element: 


$$
D_{4}=\left\{d_{1}\right\}
$$

Making this decision requires the information of the values of the following operational parameters:

1. Probability $p_{i j}^{d_{k}}$ of state transition, i.e.: $p_{42}^{(1)}=1$,

2. Conditional expected value $m_{i j}^{d_{k}}$ of time interval $T_{i j}^{d_{k}}$ of unplanned service forced by damage, i.e.: $m_{42}^{(1)}$,

3. Standard deviation $\sigma_{i j}^{d_{k}}$ of the conditional time interval of unplanned service forced by damage, i.e.: $\sigma_{42}^{(1)}$,

4. Average "profit" $r_{i j}^{d_{k}}$ gained in time unit of unplanned service forced by damage, i.e.: $r_{42}^{(1)}$,

5. Constant "profit" $b_{i j}^{d_{k}}$ (bonus) obtained from single realisation of state $e_{4}$, i.e.: $b_{42}^{(1)}$.

The distribution parameters of conditional time interval of state $\mathrm{e}$, which are necessary for making decision $\mathrm{d} 1$, should be calculated from formulas (32). Therefore, we should calculate:

6. Distribution parameters $\alpha_{i j}^{d_{k}}$ and $\lambda_{i j}^{d_{k}}$ of the conditional time interval of the service forced by damage, i.e.: $\alpha_{42}^{(1)}, \lambda_{42}^{(1)}$.

After calculating the values of the above parameters of the operation process $\{Y(t): t \geq 0\}$ for an arbitrary USO, the procedure proposed in the article (formulas $17 \div 25$ ) can be applied to determine the optimal strategy.

\section{FINAL CONCLUSIONS AND REMARKS}

Decision-making based control of the operation process of an arbitrary marine power plant device (USO), in particular the main engine (SG), is the most essential and, simultaneously, most difficult issue in operation of these devices. This results from the fact that the control is executed in a stochastic decision-making situation resulting from random conditions in which these devices operate. As a consequence, rational control of these processes requires developing stochastic decision-making models.

The article shows that these models can be developed based on the theory of semi-Markov decision-making (control) processes. To enable making operating decision which will ensure a rational course of the USO process, the model of this process is proposed in the form of a four-state semi-Markov decision-making (control) process $\{Y(t): t \geq 0\}$.

The optimisation criterion adopted when making operating decisions is the expected profit to be gained during the operation of each USO in given time interval.

The optimal decision-making strategy for the process $\{Y(t): t \geq 0\}$ is determined using profit as the optimisation criterion and the dynamic programming method making use of the well-known Bellman's principle of optimality. The developed procedure to determine the optimal strategy is as follows:

1. For each $z_{i} \in Z$ and $d_{k} \in D_{i}$, the profit $U_{i}^{d_{k}}$ is to be calculated from formula (14), which is the expected profit to be gained in a single time interval of state $z_{i}$ realisation when the decision $d_{k} \in D_{i}$ was made at the beginning of this realisation

2. For each $z_{i} \in Z$, the maximal profit $V_{z_{i}}\left(d_{1}^{*}\right)$ is to be calculated from formula (22) for the first decision $d_{1}^{*}$,

3. In successive steps, i.e. for $l=2, \ldots, m$, and for each $z_{i} \in Z$, the expected (average) profit is to be calculated from formula (21), hence for $l=2$

$$
V_{z_{i}}\left(d_{2}^{*}\right)=\max _{d_{k} \in D_{i}}\left[U_{i}^{\left(d_{k}\right)}+\sum_{\in Z} p_{i j}^{\left(d_{k}\right)} V_{z_{j}}\left(d_{1}^{*}\right)\right] .
$$

Calculating the expected (average) profit from formula (21) for the final step $(l=m)$, when at the initial time the process $\{Y(t): t \geq 0\}$ was in state $z_{i} \in Z$, is equivalent to determining the optimal strategy.

Verifying and demonstrating the practical usefulness of this model requires the use of statistical methods to estimate the probabilities $p_{i j}$ and the expectation values $E\left(T_{j}\right)$ and the parameters of the distributions of the random variables $T_{i j}$ and other performance parameters, which are considered in this article. This is possible only in the case of obtaining the realization $y(t)$ of the process $\{Y(t): t \geq 0\}$ in a sufficiently long testing interval, that is, for $t \in\left[0, t_{b}\right]$, with $t_{b} \gg 0$. Then it is possible to determine the numbers $n_{i j}(i, j=1,2,3,4 ; i \neq j)$, which denote the numbers of transitions from state $z i$ to $z j$ in a sufficiently long time depending on the decisions made.

\section{REFERENCES}

1. Bellman R., A.: Dynamic programming. Princeton Univ. Press. Princeton, New Jersey 1957.

2. Girtler J.: Limiting distribution of the three-state semiMarkov model of technical state transitions of ship power plant machines and its applicability in operating decisionmaking. Polish Maritime Research, Vol. 27, No 2(106), 2020, s. 136-144.

3. Girtler J.: Necessity for and possibility of application of the theory of semi-Markov processes to determine reliability of diagnosing systems. Journal of POLISH CIMAC, Vol. 7, No. 2 (2011), s. 45-54.

4. Girtler J.: The issue of quantum in empirical research on machines and other power systems. Journal of POLISH CIMAC, Vol. 7, No. 1 (2012), s. 57-66.

5. Girtler J.: Application of semi-Markov processes for evaluation of diesel engines reliability with regards to diagnostics. Journal of Polish CIMAC, Vol. 11, No 1, s.4753, 2017.

6. Girtler J.: Possibility of estimating the reliability of diesel engines by applying the theory of semi-Markov processes and making operational decisions by considering reliability of diagnosis on technical state of this sort of combustion engines. Combustion Engines, Vol 163, No 4. 2015, s. 67-76. 
7. Girtler J.: Usefulness of semi-Markov processes as models of the operation processes for marine main engines and other machines of ship power plants. Journal of Polish CIMAC, Vol. 9, No 2. 2014, s. 57-67.

8. Girtler J.: A semi-Markov model of fuel combustion process in a Diesel engine. Polish Maritime Research, Vol. nr S1, 2007, s.58-61.

9. Girtler J.: The semi-Markov model of energy state changes of the main marine internal combustion engine and method for evaluating its operation during ships voyage. Polish Maritime Research, Vol. 18, nr 4, 2011, s. 36-42.

10. Girtler J.: Diagnostics as a condition for control over the operation of marine internal combustion engine. Study No 28, WSM, Szczecin 1997 (in Polish).

11. Girtler J., Kuszmider S., Plewiński L.: Selected issues of operation of sea-going vessels in the aspect of navigation safety. Ed. WSM in Szczecin, Szczecin 2003 (in Polish).

12. Girtler J.: Control over operation process of marine internal combustion engines on the basis of a diagnostic decisionmaking model. AMW Scientific Journals, No. 100A, Gdynia 1989 (in Polish).

13. Grabski F.: The theory of semi-Markov operation processes of technical objects. AMW Scientific Journals, No. 75 A, Gdynia 1982 (in Polish).

14. Grabski F.: Semi-Markov models of reliability and operation. Polish Academy of Sciences, IBS. Warsaw 2002. Series: System Studies, vol. 30 (in Polish)

15. Howard R.A.: Research in semi-Markovian decision structures. J. Oper. Res. Soc. Japan, 1964, 6, nr 4, p.163-199.

16. Jewell W.S.: Markov-renewal programming. Operation Research 11, 1963, p.938-971.

17. Korczewski Z.: Operational diagnostics of marine engines. Ed. Gdansk University of Technology, Gdansk 2017 (in Polish).

18. Korczewski Z.: Endoscopy of marine engines. Acad. Ed. AMW, Gdynia 2008 (in Polish).

19. Koroluk V.S., Turbin A.F: Semi-Marcov processes and their applications. Naukowa Dumka, Kiev 1976 (in Russian).

20. Leszek W.: Empirical research. Institute of Operation Technologies, Radom 1977 (in Polish)

21. Mine H., Osaki S.: Markovian decision processes. AEPCI, Nev York 1970.
22. Pabis S.: Methodology and methods of empirical sciences. PWN, Warsaw 1985 (in Polish).

23. Rudnicki J.: Application issues of the semi-Markov reliability model. Polish Maritime Research. No 1(85)/2015, Vol. 22, pp. $55-64$.

24. Silvestrov D.S.: Semi-Marcov processes with a discrete set of states. Soviet Radio, Moscow 1980 (in Russian).

\section{CONTACT WITH THE AUTHORS}

\author{
Jerzy Girtler \\ e-mail: jgirtl@pg.edu.pl \\ Jacek Rudnicki \\ e-mail: jacekrud@pg.edu.pl \\ Gdańsk University of Technology \\ Narutowicza 11/12 \\ 80-233 Gdańsk \\ Poland
}

